\title{
PENETRAÇÃO E DISTRIBUIÇÃO DO CONSOLIDANTE PARALOID® B-72 EM CERÂMICA BRANCA ESTUDADA POR TOMOGRAFIA COM NÊUTRONS
}

\author{
Marco A. Stanojev Pereira ${ }^{a}$, Reynaldo Pugliesia,*, Marcos L. G. Andrade e Maria I. Prudêncio ${ }^{\mathrm{b}}$ \\ ${ }^{a}$ Centro do Reator de Pesquisas, Instituto de Pesquisas Energéticas e Nucleares, Av. Prof. Lineu Prestes 2242, Butantã, Cidade \\ Universitária, 05508000 São Paulo - SP, Brasil

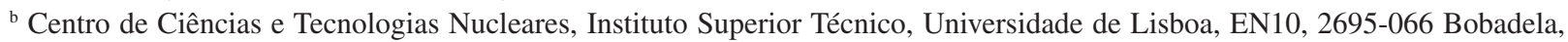 \\ Portugal
}

Recebido em 04/01/2017; aceito em 22/03/2017; publicado na web em 16/05/2017

\begin{abstract}
ASSESSMENT OF PARALOID® B-72 PENETRATION IN WHITE CERAMIC STUDIED BY NEUTRON TOMOGRAPHY. The neutron tomography technique was employed to study the penetration and distribution of the consolidant Paraloid® B-72 in ceramic. This study was carried out in white contemporary ceramic vessels which were maintained in two distinct and controlled air humidity conditions, $35 \%$ and $90 \%$, before the consolidant application (brushing technique). The results showed that the treatment with Paraloid® B-72 is more effective when applied in the drier vessel. The depth of penetration for the vessel at 35\%, reaches ( 3.3 $\pm 0.1) \mathrm{mm}$ in the wall, while for the one at $90 \%,(1.9 \pm 0.1) \mathrm{mm}$, and the maximal concentration impregnation was $26 \%$ higher. The 3D images allowed a visualization of the impregnation process, that is, its distribution, failures, homogeneity, boundary between materials. These results demonstrated the feasibility of the neutron tomography technique and of the equipment of IPEN-CNEN/SP, in support the expert work in the field of preservation and restoration of ceramic objects.
\end{abstract}

Keywords: neutron tomography; chemistry consolidant; acrylate polymers.

\section{INTRODUÇÃO}

O patrimônio cultural brasileiro é influenciado principalmente por grupos étnicos de origem indígena, europeia e africana, nos quais muitos dos objetos como ferramentas, utensílios, estatuetas, símbolos religiosos, alguns tipos de armas, ornamentos, etc., são fabricados a partir de cerâmica, madeira, fibras orgânicas, ossos e alguns metais. Especificamente a cerâmica branca, denominada na língua Tupi como "tabatinga", é classificada como Louça, designação dada a objetos cerâmicos que apresentam estruturas mais porosas, e muitos destes de interesse do nosso patrimônio histórico e cultural. ${ }^{1}$ Atualmente, os seus principais usos são na fabricação de xícaras, canecas e peças decorativas. ${ }^{2,3}$ Normalmente ao estudar objetos cerâmicos, os restauradores e especialistas procuram conhecer o seu estado de conservação e se apresentam processos de decomposição ou fragmentação. ${ }^{4,5}$ No caso de apresentarem alguma degradação, geralmente fazem uso de substâncias orgânicas específicas, conhecidas como consolidantes, que são aplicados às suas superfícies externas. Estas substâncias são polímeros acrílicos, comercialmente conhecidos como Paraloid ${ }^{\circledR}$ B-72, B-67 e B-66, Elvacite, Acrilem e, dentre estes, o primeiro é um dos mais empregados. ${ }^{6}$ Estes consolidantes atuam no objeto cerâmico formando uma película protetora em sua superfície e penetram pelos seus poros, sem no entanto selá-los completamente, deixando sua estrutura estável e permeável à umidade. ${ }^{7-9}$ Assim, a penetração e sua distribuição no objeto de interesse são fatores chave neste tipo de procedimento.

Os objetivos do presente estudo foram:

i) avaliar a penetração e a distribuição do consolidante Paraloid®

B-72 em vasos cerâmicos contemporâneos por meio da técnica de Tomografia com Nêutrons (TN). Esta técnica é muito adequada para tal propósito, uma vez que as características dos processos de interação nêutron-matéria garantem, ao mesmo tempo, uma transparência desejável do nêutron ao material cerâmico, mas

*e-mail: pugliesi@ipen.br uma elevada atenuação referente ao consolidante, pois se trata de uma substância rica em hidrogênio. ${ }^{10,11}$

ii) contribuir ao aprimoramento das técnicas de preservação, determinando por meio da TN se a penetração e a distribuição do Paraloid® B-72 no corpo cerâmico é mais efetiva, quando aplicada em condição de elevada ou de baixa umidade relativa do ar.

\section{EXPERIMENTAL}

\section{Amostras cerâmicas e o consolidante}

As amostras empregadas no presente estudo foram vasos contemporâneos fabricados em cerâmica branca, e a Figura 1 ilustra um exemplar empregado. O consolidante utilizado foi o Paraloid ${ }^{\circledR}$ B-72 (copolímero de metacrilato de etilo e acrilato de metilo com fórmula molecular genérica $\left.\left(\mathrm{C}_{21} \mathrm{H}_{25} \mathrm{ClO}_{5}\right)\right)$, um adesivo acrílico disponível comercialmente em casas especializadas em restauro, diluído em Acetona P.A. na concentração de $10 \%$ em massa, comumente empregada para este propósito. ${ }^{5,9} \mathrm{O}$ consolidante foi aplicado pela técnica de pincelagem, e somente em metade de sua parede externa.

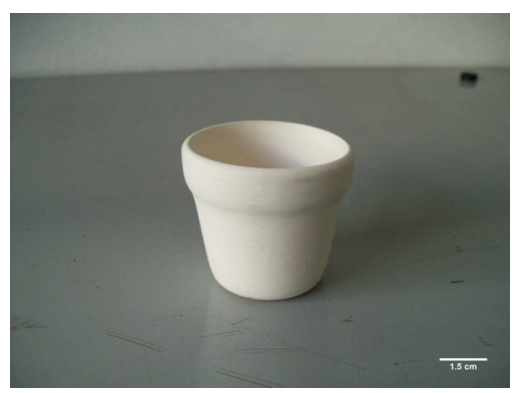

Figura 1. Tipo de vaso cerâmico branco empregado no presente estudo 


\section{Caracterização da cerâmica}

Foram utilizadas três técnicas para a caracterização da cerâmica estudada. A primeira foi a da difração de raios-X (DRX) para determinar sua composição mineralógica, e para tal propósito um dos vasos, ao qual a solução de Paraloid® B-72 não foi aplicada, foi fragmentado até um tamanho de grão de $\sim 30 \mu \mathrm{m}$, e parte do pó foi analisado em um difratômetro Rigaku (mod. Multiflex) com monocromador de grafite. A segunda foi a da Microscopia Eletrônica de Varredura (MEV) para visualizar os detalhes da superfície da cerâmica, empregando um microscópio FEI (mod. QUANTA 400 FEG). Para tal finalidade, foram inspecionados fragmentos de vasos cerâmicos sem e com a aplicação da solução de Paraloid ® B-72. A terceira técnica foi utilizada para avaliar o nível da radioatividade induzida no material cerâmico. Durante o procedimento para se obter a tomografia é importante considerar que os elementos químicos da cerâmica serão irradiados em um feixe de nêutrons intenso, e alguns poderão ser ativados. Para verificar o nível radioativo e o seu decaimento, um pedaço de cerâmica com massa de $7,5 \mathrm{~g}$ foi fragmentado até o tamanho de grão de $\sim 30 \mu \mathrm{m}$ e irradiado por $400 \mathrm{~s}$, que é o tempo de irradiação necessário para se obter uma tomografia. ${ }^{12}$ No final da irradiação, uma parte do pó com massa 0,25 $\mathrm{g}$ foi analisada em um sistema de contagem Ludlum (mod. 2929 dual scaler) sensível às radiações alfa, beta e gama, e a outra parte analisada em um espectrômetro com detector de $\mathrm{Ge}(\mathrm{Li})$, a fim de identificar os elementos químicos responsáveis pela emissão de radiação gama. ${ }^{4}$

\section{Estudo da penetração e da distribuição do Paraloid® B-72}

Como mencionado anteriormente, a técnica empregada foi a da tomografia com nêutrons. O equipamento está instalado no canal de irradiação (BH) 14 do Reator Nuclear de Pesquisas IEA-R1 do Instituto de Pesquisas Energéticas e Nucleares (IPEN/CNEN-SP), um reator do tipo piscina que opera à potência de $5 \mathrm{MW}$. O feixe de nêutrons na posição de irradiação das amostras é de $8 \times 10^{6} \mathrm{n} \mathrm{s}^{-1}$ $\mathrm{cm}^{-2}$, com um diâmetro máximo de $16 \mathrm{~cm}$. A Figura 2 mostra um diagrama esquemático deste equipamento. ${ }^{12,13}$ Uma tomografia é obtida da seguinte forma: o vaso a ser irradiado é posicionado numa mesa giratória motorizada; a intensidade de nêutrons transmitida pelo vaso incide em uma tela cintiladora de Fluoreto de Lítio ( ${ }^{6} \mathrm{LiF}(\mathrm{ZnS})$ $18 \times 24 \mathrm{~cm}$ ) formando uma imagem bidimensional de sua estrutura interna; um espelho plano reflete esta imagem para uma câmera de vídeo digital (ANDOR iKon-M), onde é capturada e, em seguida, armazenada num computador; devido à baixa intensidade da luz gerada pela tela, o cintilador, o espelho e a câmera são instalados no interior de uma caixa vedada contra luz externa; uma interface eletromecânica conecta a mesa e a câmera, de tal forma que, após a imagem ser capturada, a mesa gira $0,9^{\circ}$, e outra imagem é capturada até completar $360^{\circ}$. O tempo necessário para a captura de todas as imagens é de $400 \mathrm{~s}$; o arquivo das imagens armazenadas é o dado de entrada para o software Octopus V8.0 gerar o arquivo de imagens reconstruídas (tomografias), que é o dado de entrada para outro software VG Studio Max V2.2 gerar imagens 3D da estrutura interna do vaso. Ambos os softwares estão instalados em um computador tipo estação de trabalho (DELL precision 5500). ${ }^{12,13}$

\section{RESULTADOS E DISCUSSÕES}

O resultado de (DRX) mostrou que a composição mineralógica da cerâmica é quartzo $\left(\mathrm{SiO}_{2}\right)(60-70 \%)$ e gehlenita $\left(\mathrm{Ca}_{2} \mathrm{Al}(\mathrm{SiAl}) \mathrm{O}_{7}\right)$ (32-40\%), muito comuns neste tipo de cerâmica. Também foram encontradas pequenas quantidades de periclase $(\mathrm{MgO})(\sim 3 \%)$ e anortita $\left(\mathrm{CaAl}_{2} \mathrm{Si}_{2} \mathrm{O}_{8}\right)(\sim 3 \%)$. Esta composição mineral indica uma elevada temperatura de queima. ${ }^{5,7}$

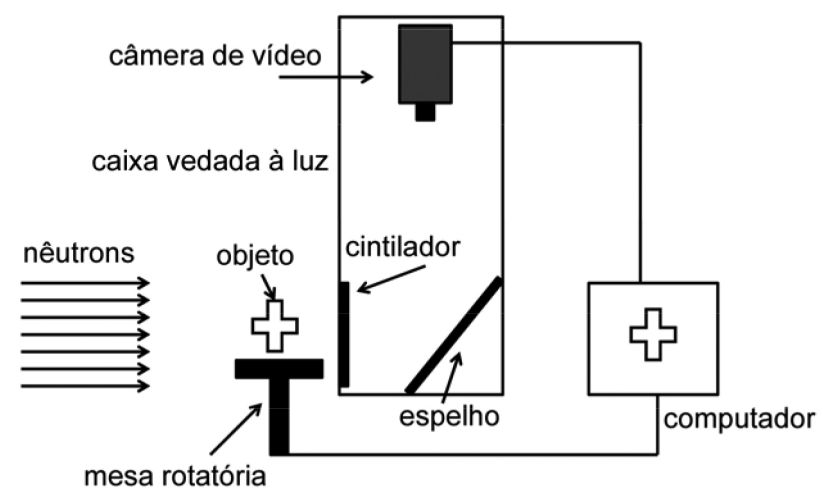

Figura 2. Diagrama esquemático do equipamento para tomografia com nêutrons

As imagens obtidas pelo MEV estão mostradas nas Figuras 3. $\mathrm{O}$ fragmento sem consolidante (Figura $3 \mathrm{a}$ ) exibe poros e vazios com grande variação de dimensões, enquanto que o outro, com consolidante (Figura 3b), exibe uma agregação não homogênea da solução com os poros e vazios parcialmente selados, o que mantêm a permeabilidade de sua superfície..$^{5,8,9}$
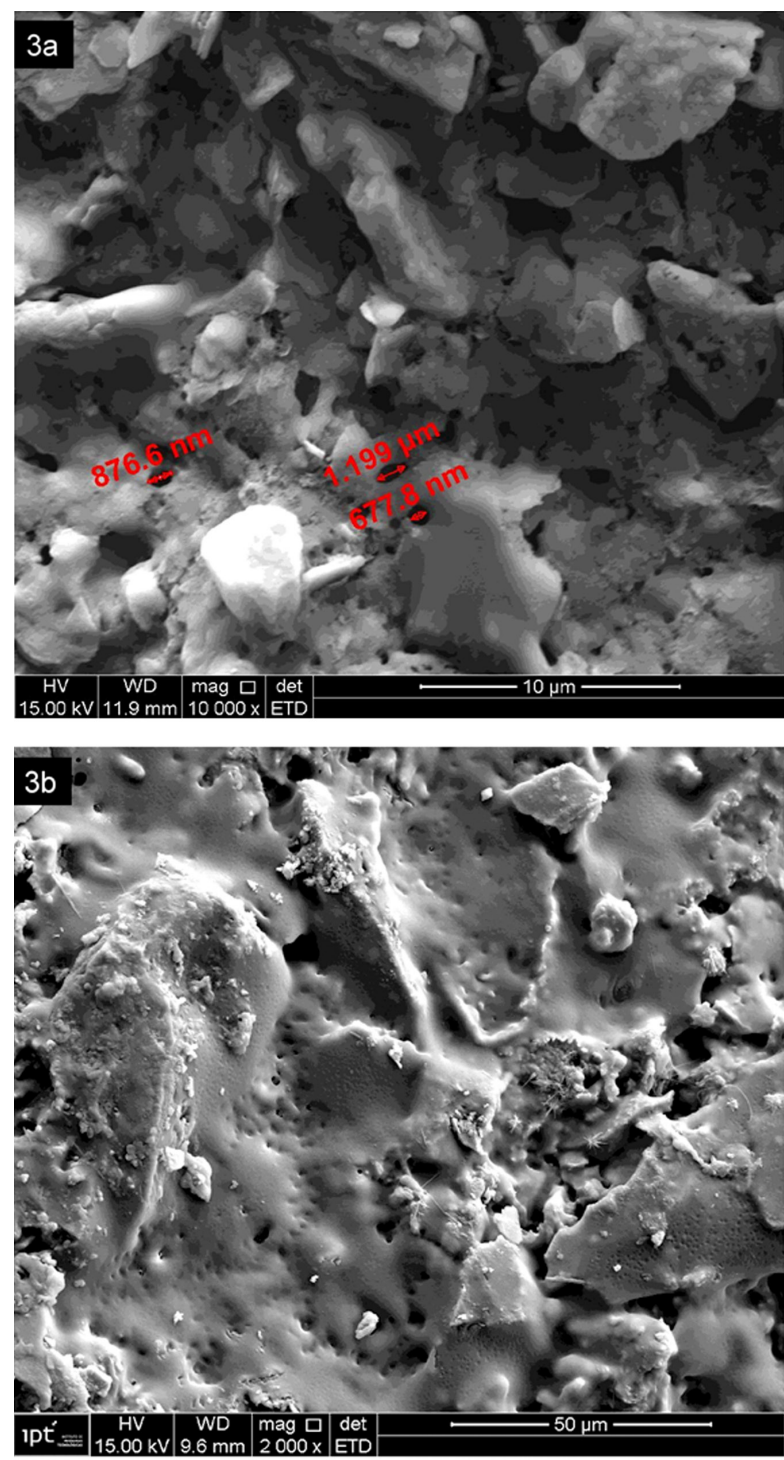

Figura 3. Imagens da superfície da cerâmica: sem (3a) e com (3b) Paraloid ${ }^{\circledR}$ $B-72$ 
Com relação à radioatividade induzida no pó cerâmico irradiado no feixe de nêutrons do equipamento para tomografia, os resultados obtidos mostraram que após 24 horas do término da irradiação, a atividade total (contagens/segundo) causada pelas radiações alfa, beta e gama decai ao nível da radiação de fundo do laboratório onde esta medida foi realizada. ${ }^{14}$ Os resultados da análise por ativação neutrônica revelaram que os elementos químicos responsáveis pela intensidade da radiação gama induzida são o magnésio e o alumínio, com meias-vidas de 9 minutos e 2,3 minutos, respectivamente, que estão presentes na gehlenita, periclase e anortita. ${ }^{15}$

O estudo da penetração e distribuição do Paraloid ${ }^{\circledR}$ B-72, por tomografia com nêutrons, foi realizado em dois vasos cerâmicos. O primeiro foi mantido durante 5 horas em uma estufa à temperatura de $70^{\circ} \mathrm{C}$, e em seguida permaneceu por sete dias em um dessecador à pressão atmosférica, num ambiente onde a umidade relativa do ar era de 35\%. Após este período, a solução de Paraloid® B-72 foi aplicada com um pincel em parte de sua superfície externa, e foi deixado à temperatura ambiente $\left(25^{\circ} \mathrm{C}\right)$ durante $\sim 3$ horas para a secagem da acetona. O segundo permaneceu por sete dias em um outro dessecador à pressão atmosférica e a $90 \%$ de umidade. A aplicação do Paraloid® B-72 e a secagem seguiram os mesmos procedimentos aplicados ao vaso anterior. A penetração e a distribuição da solução de Paraloid® B-72 no vaso cerâmico foi estudada a partir das tomografias referentes ao plano $\mathrm{XY}$, que consistem em um arquivo de computador com 165 imagens ou tomos, cada uma representando uma espessura de $0,28 \mathrm{~mm}$ do vaso inspecionado. A Figura 4 mostra uma destas fatias para o vaso mantido à umidade de $35 \%$, na qual o Paraloid® B-72 se destaca como um anel semicircular brilhante à esquerda (local onde a solução foi aplicada), enquanto que o corpo cerâmico sem o consolidante aparece mais escuro à direita.

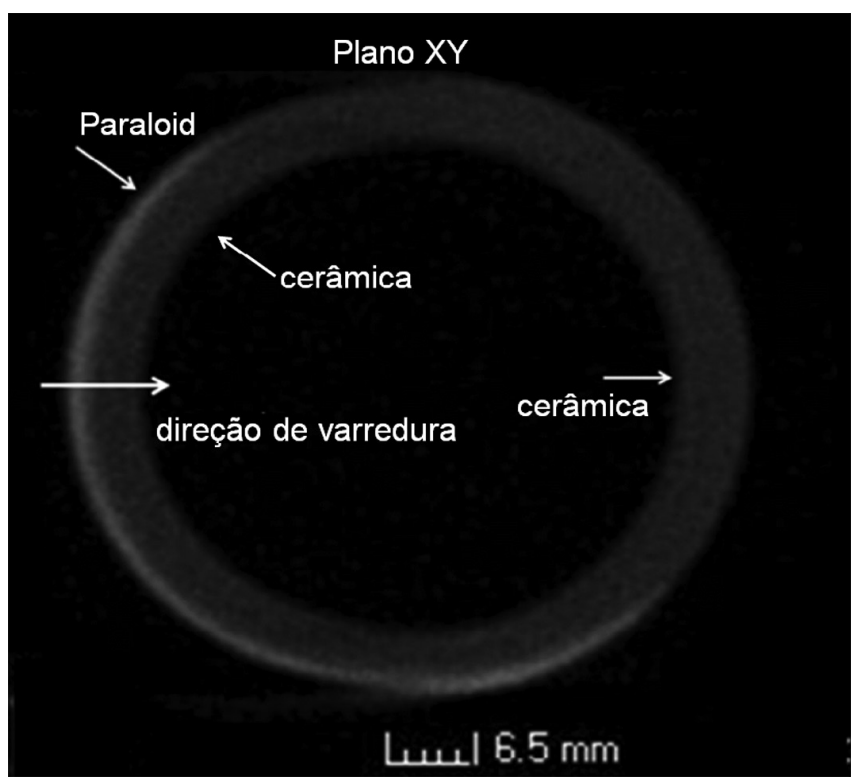

Figura 4. Tomografia (plano XY) para o vaso mantido à umidade de $35 \%$

A quantificação da penetração foi realizada a partir da determinação da distribuição da intensidade dos Níveis de Cinza (NC) em função da coordenada de varredura " $x$ " através da espessura da parede do vaso, conforme mostrado na Figura 4. Uma vez que as tomografias são imagens de 8 bits, as intensidades dos níveis de cinza variam de 0 (região mais escura) à 255 (região mais clara). ${ }^{12}$ Conforme mencionado anteriormente, as Figuras $3 \mathrm{a}$ e $3 \mathrm{~b}$ mostram poros e vazios com grande variação de dimensões. Com a finalidade de levar em conta a possível influência destes parâmetros na penetração e na distribuição do consolidante na parede do vaso, foram avaliadas dez distribuições correspondentes à dez fatias sequenciais do arquivo de imagens reconstruídas, ao longo do plano XY. Uma distribuição típica para o vaso mantido à umidade de $35 \%$ é mostrada na Figura 5. Para cada distribuição foram avaliados os seguintes parâmetros: profundidade da penetração ( $\overline{\mathrm{AC}}$ eixo "X"); profundidade para a qual a concentração de Paraloid $\AA$ B-72 é máxima ( $\overline{\mathrm{AB}}$ eixo "X"); espessura da parede do vaso ( $\overline{\mathrm{AD}}$ eixo "X”); concentração máxima da impregnação dada pelo valor máximo de $\mathrm{NC}(\overline{\mathrm{OB}}$ eixo "Y"). Os resultados mostrados na Tabela 1 são os valores médios com suas respectivas incertezas, obtidos das 10 distribuições estudadas.

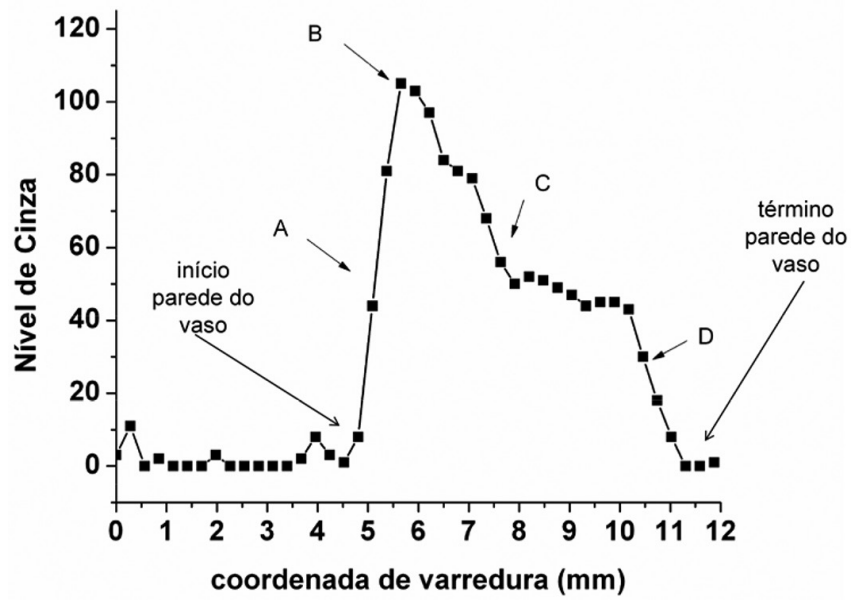

Figura 5. Distribuição típica dos níveis de cinza, ao longo da parede do vaso (da borda para o seu interior) mantido à umidade de $35 \%$

Para o vaso mantido à umidade de $90 \%$, a irradiação foi realizada seguindo os mesmos procedimentos empregados para o mantido a $35 \%$, e uma distribuição típica é mostrada na Figura 6. A Tabela 1 resume todos os valores obtidos para a penetração e distribuição do Paraloid ${ }^{\circledR}$ B-72 para ambos os vasos estudados.

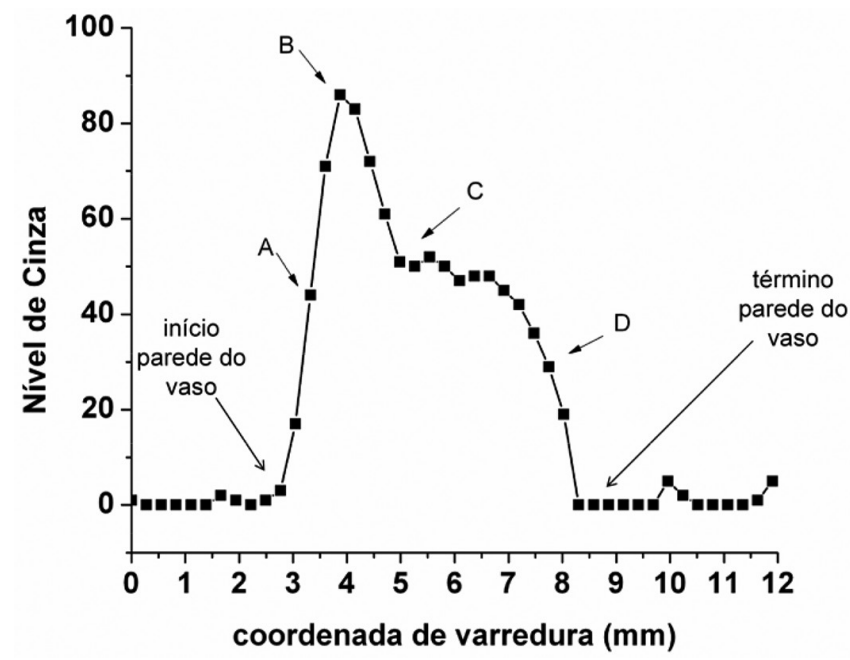

Figura 6. Distribuição típica dos níveis de cinza, ao longo da parede do vaso (da borda para o interior) mantido à umidade de $90 \%$

Com o intuito de complementar o estudo realizado, na Figura 7 estão apresentadas algumas imagens 3D da estrutura interna de um dos vasos tomografados, obtidas por meio do software VGStudio Max 2.2. Estas imagens referem-se ao vaso que foi mantido na umidade de $35 \%$ e permitiram uma visualização macroscópica da distribuição do Paraloid® B-72 em suas paredes, e da profundidade de penetração. ${ }^{6}$ A Figura 7a mostra a imagem do vaso íntegro; a Figura 7b evidencia 
Tabela 1. Resultados obtidos para a penetração e distribuição do Paraloid® B-72 nos vasos mantidos às umidades de $35 \%$ e $90 \%$

\begin{tabular}{lcc}
\hline Parâmetro & Valor médio (35\%) & Valor médio (90\%) \\
\hline $\begin{array}{l}\text { Profundidade de } \\
\text { penetração (mm) }\end{array}$ & $3.3 \pm 0.1$ & $1.9 \pm 0.1$ \\
$\begin{array}{l}\text { Profundidade de máxima } \\
\text { concentração (mm) }\end{array}$ & $0.77 \pm 0.01$ & $0.67 \pm 0.02$ \\
$\begin{array}{l}\text { Espessura da parede } \\
(\text { mm) }\end{array}$ & $5.3 \pm 0.1$ & $4.6 \pm 0.1$ \\
Máximo valor de NC & $112 \pm 2$ & $89 \pm 1$ \\
\hline
\end{tabular}
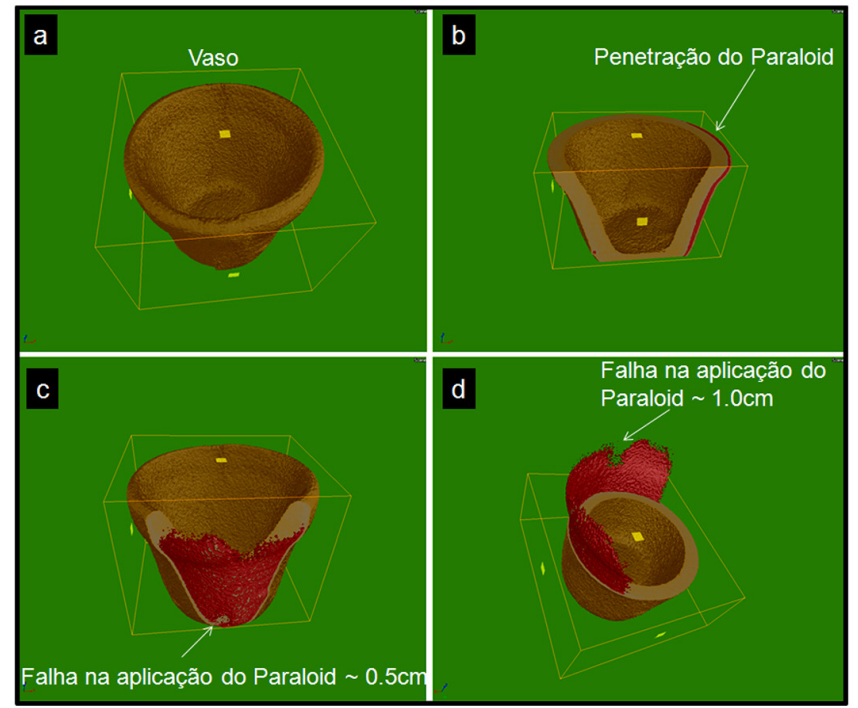

Figura 7. Imagens $3 D$ da distribuição do Paraloid ${ }^{\circledR}$ B-72 para o vaso mantido à umidade de $35 \%$

partes internas do material cerâmico, da qual foram removidas com as ferramentas de processamento do próprio software o topo e o lado frontal, de forma a permitir a visualização da profundidade de penetração do Paraloid® B-72 (detalhe em vermelho), bem como a fronteira cerâmica/Paraloid; as Figuras 7c e 7d expõem partes do corpo cerâmico e do consolidante no interior da cerâmica, o que permitiu a visualização de duas falhas (indicadas por setas) com tamanho considerável, do processo de impregnação do consolidante.

\section{CONCLUSÕES}

O presente trabalho visou avaliar, mediante a técnica de tomografia com nêutrons, a penetração e a distribuição do consolidante Paraloid® B-72, diluído em acetona a $10 \%$ em massa, em vasos de cerâmica branca contemporânea, mantidos em duas condições extremas de umidade relativa do ar, um a $35 \%$ e outro a $90 \%$, antes de sua aplicação.

De acordo com os resultados das distribuições dos níveis de cinza nas Figuras 5 e 6, a concentração de Paraloid® B-72, para ambos os vasos analisados, aumenta muito rapidamente próximo à superfície na qual foi aplicada, atinge seu máximo muito próximo dessa superfície e diminui à medida que a espessura da parede aumenta. Além disto, os dados da Tabela 1 mostram que tanto a profundidade de penetração quanto o valor máximo de $\mathrm{NC}$, que é proporcional à concentração do consolidante na cerâmica, são maiores para o vaso mantido a $35 \%$, o que significa que na condição mais seca o processo de impregnação é mais eficiente. Desta forma, é recomendado um pré tratamento térmico para a secagem de amostras encontradas em sítios arqueológicos, ou que já estejam armazenadas em depósitos, situados em regiões de umidade elevada. Para finalizar, é importante mencionar que o conjunto dos dados obtidos mostrou que a impregnação do Paraloid ${ }^{\circledR}$ B-72 por pincelagem pode ser não homogenea em toda a extensão da parede onde foi aplicado, e esta não homogeneidade pôde ser claramente observada nas imagens 3D apresentadas.

O intervalo de tempo para o qual a radioatividade induzida pelos nêutrons na cerâmica se torna insignificante é de cerca de 24 horas a partir do instante final do término da tomografia. No entanto, é muito importante enfatizar que este resultado é válido apenas para esta cerâmica específica, pois, para outras cerâmicas, tanto as composições básicas como as impurezas podem levar a diferentes níveis de radiação e a intervalos de tempo de decaimento.

Os resultados demonstraram a viabilidade da técnica da tomografia com nêutrons e do equipamento do IPEN-CNEN/SP, em apoio ao trabalho de especialistas no campo da preservação e restauração de objetos cerâmicos. Outros importantes resultados obtidos anteriormente, referentes à visualização e à determinação das dimensões de trincas e de descontinuidades em outros corpos cerâmicos, utilizando esta técnica, reforçam a sua utilização para este propósito. ${ }^{5,12}$

\section{AGRADECIMENTOS}

Os autores vinculados ao IPEN-CNEN/SP agradecem à Agência Internacional de Energia Atômica - A.I.E.A. e ao Conselho Nacional de Desenvolvimento Científico e Tecnológico - CNPq pelos suportes financeiros ao projeto CRP cod. F11018 e pela bolsa de pós-doutorado 114862/2015-0, respectivamente.

O autor vinculado ao C2TN/IST agradece ao FCT (Fundação para Ciência e Tecnologia) o apoio através do projeto UID/ Multi/04349/2013.

\section{REFERÊNCIAS}

1. Gonçalves Dias, A. Em Dicionário da língua Tupi chamada Língua Geral dos Índios do Brasil; Brockhaus, F. A., ed.; Lipsia, 1858, pg. 162.

2. Motta, J. F. M.; Zanardo, A.; Cabral Jr., M.; Cerâmica Industrial 2001, $6,28$.

3. Gorea, M.; Benea, M.; Studia UBB Geologia 2002, XLVII, 77.

4. IAEA Radiation Technology Series No. 2. Nuclear Techniques for Cultural Heritage Research, 2011, pp. 4, 133.

5. Prudêncio, M. I.; Stanojev Pereira, M. A.; Marques, J. G.; Dias, M. I.; Esteves, L.; Burbidge, C. I.; Trindade, M. J.; Albuquerque, M. B.; J. Archaeol. Sci. 2012, 39, 964.

6. Constâncio, C.; Franco, L.; Russo, A.; Anjinho, C.; Pires, J.; Vaz, M. F.; Carvalho, A. P.; J. Appl. Polym. 2010, 116, 2833.

7. Vaz, M. F.; Pires, J.; Carvalho, A. P.; Journal of Cultural Heritage 2008, 9, 269.

8. Fleischer, G.; Nimmrichter, J.; Rohatsch, A.; Geophysical Research Abstracts 2005, 7 (SRef-ID: 1607e7962/gra/EGU05-A-04479).

9. Carreti, E.; Dei, L.; Prog. Org. Coat. 2004, 49, 282.

10. Huges, D. J.; Harvey, J. A.; Neutron cross section, McGrall-Hill: New York, 1955

11. Rant, J.; Milic, Z.; Istenic, J.; Knific, T.; Lengar, I.; Rant, A.; Appl. Radiat. Isot. 2006, 64, 7.

12. Schoueri, R.; Domienikan, C.; Toledo, F.; Andrade, M. L. G.; Stanojev Pereira, M. A.; Pugliesi, R.; Appl. Radiat. Isot. 2014, 84, 22.

13. Stanojev Pereira, M. A.; Schoueri, R.; Domienikan, C.; Toledo, F.; Andrade, M. L. G.; Pugliesi, R.; Appl. Radiat. Isot. 2013, 75, 6.

14. http://nvlpubs.nist.gov/nistpubs/Legacy/TN/nbstechnicalnote1118.pdf, acessada em Abril 2017.

15. http://nucleardata.nuclear.lu.se/toi/perchart.htm, acessada em Abril 2017. 